\title{
AN ON-LINE AND ROBUST IDENTIFICATION SCHEME FOR TIME- VARYING ARMA PROCESSES
}

\author{
Mehmet Önder Efe ${ }^{1}$, Okyay Kaynak ${ }^{2}$ and Bogdan M. Wilamowski ${ }^{3}$ \\ ${ }^{1}$ The Ohio State University, Electrical Engineering Department \\ Columbus, OH 43210, U.S.A., onderefe@ee.eng.ohio-state.edu \\ ${ }^{2}$ Bogazici University, Electrical and Electronic Engineering Department \\ Bebek, 80815, Istanbul, Turkey, kaynak@boun.edu.tr \\ ${ }^{3}$ University of Idaho, Graduate Center at Boise \\ 800 Park Blvd., Boise, ID 83712, U.S.A., wilam@ieee.org
}

\begin{abstract}
In this paper, a novel method for extracting the values of the coefficients of time-varying ARMA processes is proposed. The approach discussed assumes solely that the orders of the numerator and the denominator polynomials are known. The algorithm is demonstrated to be stable in the sense of Lyapunov, furthermore, it is shown in the paper that the evolution in the parameter space takes place in a finite volume. The proposed method is cost effective and is based on the variable structure systems theory, which is well known with its robustness to uncertainties. In the simulation example, the coefficients of a second order ARMA process is extracted by the use of the algorithm presented.
\end{abstract}

\section{INTRODUCTION}

Identification of systems having uncertainties and impreciseness constitutes a central part in the practice of systems engineering. This fact is intimately related to the desire for constructing systems having a degree of autonomy enabling the system to operate in changing environmental conditions. One way of handling the difficulties stemming from the uncertainties is to utilize a suitable identification method to collect as much information as possible in an organized fashion. In the literature, most widely used approaches for system identification are based on Least Mean Squares (LMS), Recursive Least Squares (RLS), Gradient Descent (GD), Levenberg-Marquardt method (LM) or their variants [15]. The prime difficulties in implementing these algorithms are the necessity to costly hardware for data storage, high sensitivity to changes in the input signal, getting stuck to local minima or the need for matrix inversions at some intermediate stages. Depending on the nature of the problem in hand, the designer is expected to choose the most appropriate adjustment technique leading to high performance with low cost. Apparently, the design of such a parameter tuning scheme is a challenge even for the simple tasks. Among many alternatives existing in the literature for parameter tuning, once the structure of the ARMA process is chosen, the designer is faced to two performance measures, namely, the speed of adaptation and the accuracy of the realization after adaptation, which are intimately related to the adopted tuning strategy [3].

Another important feature of an adaptation mechanism is the robustness, which can be defined as the capability of compensating the uncertainties and the capability of keeping the cost measure at the lowest level under the presence of parameter variations in the process to be identified. If one defines the discrepancy between the output of a time-varying ARMA structure and that of an identifier ARMA structure as the error measure, the task of maintaining the zero output error with changing parameters clearly implies the need to a robustness in the tuning mechanism.

One way of designing a robust identification scheme is to utilize Variable Structure Systems (VSS) theory in constructing the adaptation mechanism [6-7]. VSS theory is well known with its robustness to uncertainties and the use of this theory introduces certain invariance properties in a predefined subspace of the state space defined by the error and its several time derivatives. Various applications utilizing VSS theory have appeared in the literature, which particularly focus on the robust control of nonlinear systems [6-9]. In order to understand the use of a technique of control engineering expertise for identification purposes, it is beneficial to dwell on what the framework prescribes in the field of control briefly.

For a system of order $l$, the decision dynamics in the state space is characterized by an (l-1)-dimensional hypersurface passing through the origin. Using the sign of a quantity describing the location of the hypersurface, two modes of motion can be created namely the mode lasting until a hitting to the hypersurface occurs, and the mode on the hypersurface, which is called sliding surface. In the literature, the former is called reaching mode while the latter is named sliding mode and the control theory uses the term Sliding Mode Control (SMC) due to the latter dynamic behavior. An important property of the SMC design is that the trajectories in the phase space are all directed towards the sliding surface, furthermore, once the error vector starts lying on this hypersurface, it directly slides towards the origin because of the description of the hypersurface. The reason for this is not only the fact that the hypersurface itself is a location in the phase space but also it describes a particular dynamics. 
In using VSS theory for identifying ARMA processes, one should consider the diagram in Figure 1. The two structures are excited by the same signal $(x)$ and the discrepancy between the produced outputs $\left(s_{c}\right)$ is used as the error measure. This quantity is then processed in the tuning strategy and the corresponding parameter values (p) are sent to the identifier and the next output is produced with the new parameter vector. Since the use of such identification techniques cover a wide spectrum including nonlinear control, signal processing and biomedical applications, the method discussed in this paper is of substantial importance due to its computational advantages and robustness [5,10-12].

In the second section, the structure of the ARMA process to be identified and the structure of the identifier are described and the proposed tuning strategy is analyzed in detail. The third section presents the simulations performed and the conclusions are presented at the end of the paper.

\section{STRUCTURE OF THE ARMA PROCESS AND THE PARAMETER TUNING STRATEGY}

Consider the delay system described by (1).

$$
y(t)=-\sum_{k=1}^{N} a_{k} y(t-k T)+\sum_{k=0}^{M} b_{k} x(t-k T)
$$

where $M$ and $N$ define the delay depth in input $(x)$ and output (y), and $T$ is the constant delay time parameter. The system above can be represented in s-domain as given in (2) and, and by direct substitution of $z=e^{s T}$ the z-domain equivalent is obtained as given in (3).

$$
\begin{gathered}
H(s)=\frac{Y(s)}{X(s)}=\frac{\sum_{k=0}^{M} b_{k} e^{-k T s}}{1+\sum_{k=1}^{N} a_{k} e^{-k T s}} \\
H(z)=\frac{Y(z)}{X(z)}=\frac{\sum_{k=0}^{M} b_{k} z^{-k}}{1+\sum_{k=1}^{N} a_{k} z^{-k}}
\end{gathered}
$$

In above, the sampling period is assumed to be equal to $T$. The system given in (1) with $y(t)=0$ for $t \leq 0$ and the transfer function representation in (2) produce the same outputs in response to the same excitation signals. When the response is compared with that obtained from (3) at integer multiples of delay time $\mathrm{T}$, it should be obvious that the ARMA process in (3) is going to respond what is observed from the continuous time equivalent of (1).

The representations given in (1) through (3) has some parameters denoted by $a_{k}$ and $b_{k}$ and these parameters are assumed to be unknown. The transfer function representation given in (3) can be expressed as a difference equation as given in (4) with $n$ being the discrete time index.
$y[n]=-\sum_{k=1}^{N} a_{k} y[n-k]+\sum_{k=1}^{M} b_{k} x[n-k]$

More compactly, the parameter vector and the regression vector can be described as in (3) and (4) respectively.

$$
\begin{aligned}
& \underline{p}=\left[\begin{array}{llllllll}
a_{1} & a_{2} & \cdots & a_{N} & b_{0} & b_{1} & \cdots & b_{M}
\end{array}\right]^{T}
\end{aligned}
$$

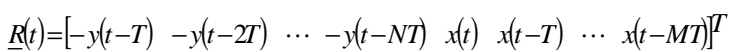

The input/output relationship in (1) can be rewritten as follows:

$$
y(t)=\underline{p}^{T}(t) \underline{R}(t)
$$

In order not to be in conflict with the physical reality, the designer must impose the following inequalities, the truth of which state that the parameters of the process $(\underline{p})$, the time derivative of the regression vector $(\underline{R})$ and the time derivative of the desired output of the continuous process $\left(y_{d}\right)$ remain bounded.

$$
\begin{aligned}
& \|\underline{p}\|=\sqrt{\underline{p}^{T} \underline{p}} \leq B_{p} \\
& \|\underline{\dot{R}}\| \leq B_{\dot{R}} \\
& \left\|\dot{y}_{d}\right\| \leq B_{\dot{y}_{d}}
\end{aligned}
$$

Theorem 2.1. The adaptation of process parameters as described in (11) enforces the process coefficients to values resulting in zero learning error level in one dimensional phase space, whose argument is defined as $s_{c}=y-y_{d}$.

$$
\underline{\dot{p}}=-\frac{\underline{R}}{\underline{R}^{T} \underline{R}} k \operatorname{sgn}\left(s_{c}\right)
$$

where, $K$ is a sufficiently large positive constant satisfying (12).

$$
k>B_{p} B_{\dot{R}}+B_{\dot{y}_{d}}
$$

The adaptation mechanism in (11) drives an arbitrary initial value of $s_{c}$ to zero in finite time denoted by $t_{h}$ satisfying the inequality in (13).

$t_{h} \leq \frac{\left|s_{c}(0)\right|}{k-\left(B_{p} B_{\dot{R}}+B_{\dot{y}_{d}}\right)}$

Theorem 2.2. If the identification scheme enters the sliding mode $s_{c}=0$ and remains in it thereafter, then the parameters of the identifier, $\underline{p}$, evolve bounded. 


\section{SIMULATION EXAMPLE}

In the simulations, we assume that the identification scheme observes the system output, the desired output and the regression vector at discrete time instants, i.e. the adaptation rule of (11) takes the following form.

$$
\underline{\dot{p}}=-\frac{\underline{R}}{\underline{R}^{T}} \underline{R} \operatorname{sggn}\left(s_{c}\right) \text { when } k T \leq t<(k+1) T
$$

In the examples, an ARMA process structure described below is considered for identification. Apparently, the output of this system is what the identifier must realize by appropriately tuning its parameters.

$$
y_{d}[n]=-\sum_{k=1}^{2} a_{k} y_{d}[n-k]+\sum_{k=0}^{2} b_{k} x[n-k]
$$

It is assumed that the coefficients of the above structure are changing in time. In Table 1, the target values of the coefficients to be extracted are given. It should be clear from the table that there are four different ARMA structures, each one of which becomes active during a certain interval of 40 seconds. More explicitly, for the first 40 seconds, i.e. $r=1$, the ARMA structure is characterized by the difference equation in (32), then a 5 seconds of transition phase takes place and during the following 40 seconds, i.e. $r=2$, the process is changed smoothly to that in (33). In (34) and (35), the structures activated in the next two intervals are described.

$$
\begin{aligned}
y_{d 1}[n]= & 0.9 y_{d 1}[n-1]-0.2 y_{d 1}[n-2]+x[n]+0.9 x[n-1] \\
y_{d 2}[n]= & y_{d 2}[n-1]+0.5 y_{d 2}[n-2]+ \\
& x[n]-0.25 x[n-1]-0.375 x[n-2] \\
y_{d 3}[n]= & -0.36 y_{d 3}[n-2]+x[n]-1.3 x[n-1]+0.42 x[n-2] \\
y_{d 4}[n]= & -x[n]+0.25 x[n-1]+1.5 x[n-2]
\end{aligned}
$$

The change of structures between the successive intervals is performed by suitably combining the all four ARMA structures. For instance, the transition between the time instants 40 and 45 seconds is illustrated in Figure 2. A weight is gradually activating the structure in (33) by multiplying its output with $w$ and the effect of the structure in (32) is decreasing since its multiplier is 1-w. This enables us to observe a soft switching between different ARMA processes. In the transition regions, the mixed process possesses fourth order numerator and denominator. The corresponding coefficients can be evaluated as given in (36) through (44). The second subscripts are used to specify the interval number.

$$
\begin{aligned}
\tilde{b}_{0}= & (1-w) b_{0, r}+w b_{0, r+1} \\
\tilde{b}_{1}= & (1-w)\left(b_{0, r} a_{1, r+1}+b_{1, r}\right)+w\left(b_{0, r+1} a_{1, r}+b_{1, r+1}\right) \\
\tilde{b}_{2}= & (1-w)\left(b_{1, r} a_{1, r+1}+b_{0, r} a_{2, r+1}+a_{2, r}\right) \\
& +w\left(b_{1, r+1} a_{1, r}+b_{0, r+1} a_{2, r}+a_{2, r+1}\right)
\end{aligned}
$$

$$
\begin{aligned}
& \tilde{b}_{3}=a_{1, r} a_{2, r+1}+a_{1, r+1} a_{2, r} \\
& \tilde{b}_{4}=1 \\
& \tilde{a}_{1}=a_{1, r}+a_{1, r+1} \\
& \tilde{a}_{2}=a_{2, r}+a_{2, r+1}+a_{1, r} a_{1, r+1} \\
& \tilde{a}_{3}=a_{1, r} a_{2, r+1}+a_{2, r} a_{1, r+1} \\
& \tilde{a}_{4}=a_{2, r} a_{2, r+1}
\end{aligned}
$$

In the example considered a Gaussian distributed random signal having zero mean and variance equal to unity is used to excite the process to be identified and the identifier. The aim is to match the two output signals in time domain. For this purpose, the error $\left(s_{c}\right)$ is evaluated and used in the adjustment mechanism of (30). The sampling rate $(T)$ has been set to $1 \mathrm{msec}$, and the uncertainty bound parameter $K$ has been set to 100 . Initially, the parameters of the identifier are all set to zero.

It should here be noted that the uncertainty bound parameter denoted by $K$ is a multiplier of the dynamic adaptation law of (30). Apparently, the small values of $K$ will decrease the speed of learning and large values will increase. However, since $K$ is a parameter describing the mobility of the parameters, its extreme values can cause instabilities. Unlike its lower bound described in (12), the upper bound is determined by the physical constraints.

The extracted values of the parameters of the numerator polynomial are depicted in Figure 3 and those of the denominator polynomial are illustrated in Figure 4. If one compares the values estimated by the identifier with those given in Table 1, it is seen that the correct values are found very quickly and very accurately. Especially the during transition intervals [40 secs., 45 secs.], [85 secs., 90 secs.] and [130 secs., 135 secs.], the process to be identified becomes a fourth order one, which is characterized by (36)-(44), and the strategy proposed quickly converges when the order of the structure becomes equal to that of the identifier. In Figure 4, the pole-zero plots of the four different structures are illustrated. It can directly be inferred from this figure that the system under investigation is changing its characteristics radically, and this constitutes a challenge for most identification schemes.

Lastly in this section, the computational burden of the algorithm is analyzed. Unlike LMS algorithm, the method presented does not need to store some history of the system under investigation and operates on-line. Furthermore, no matrix inversion is needed throughout the operation. For the structure studied in this paper, i.e. $N=2$ and $M=2$, a single forward pass from (1) with tuning of parameters with (30) requires 46 floating point operations (flops), which is a quite promising value for real-time realizations when considered with the accurate identification performance. In Figure 6 , the required number of flops has been illustrated for varying values of $M$ and $N$. Clearly, the computational cost is not excessive but the most important aspect of the proposed approach is the behavior of the increase in complexity as the 
dimensionality of the parameter vector increases. In Figure 7 , the cost graph is given as a surface, which figures out that the complexity is $O(M+N)$, i.e. the required number of flops will increase linearly as the dimension of the parameter vector increases.

\section{CONCLUSIONS}

The lack of sufficient knowledge about a system of interest requires an in depth investigation procedure for developing mechanisms that need the parameters characterizing the behavior of the system. For this purpose, various approaches are used to identify the system parameters. However, some of these schemes suffer from the computational complexity, while the applicability of some are subject to the availability of a priori knowledge. If the goal of handling the uncertainties with high performance and low cost is considered, the use of VSS theory becomes more comprehensible.

The results obtained through some simulations have clearly stipulated that the extraction of the unknown parameters can be achieved neither by storing excessive amount of data nor by occupying the CPU oppressively.

The radical changes in the ARMA structure have appropriately been detected by the discussed algorithm and the corresponding parameter set have precisely been determined. The finite volume parameter evolution and the stability claims of the proposed technique are proved and the theoretical results have been confirmed by the example presented.

\section{ACKNOWLEDGMENTS}

This work is supported by NSF (Grant No: 9906233).

\section{REFERENCES}

[1] Ljung, L., System Identification, Prentice-Hall International, New Jersey, 1987.

[2] Haykin, S., Adaptive Filter Theory, Prentice-Hall International, New Jersey, 1991.

[3] Honig, M.L. and Messerschmitt, D.G., Adaptive Filters, Kluwer Academic Publishers, Massachussetts, U.S.A., 1984.

[4] Krishnapura, V.G. and Jutan, A., "ARMA Neuron Networks for Modeling Nonlinear Dynamical Systems," The Canadian Journal of Chemical Engineering, Vol. 75, pp.574-582, 1997.

[5] Efe, M. O. and O. Kaynak, "A Comparative Study of Soft Computing Methodologies in Identification of Robotic Manipulators", Robotics and Autonomous Systems, Vol. 30, No. 3, pp. 221-230, 2000.

[6] Efe, M. O., Variable Structure Systems Theory Based Training Strategies for Computationally Intelligent Systems, Ph.D. Thesis, Bogazici University, 2000.

[7] Efe, M. O., O. Kaynak and X. Yu, "Sliding Mode
Control of a Three Degrees of Freedom Anthropoid Robot by Driving the Controller Parameters to an Equivalent Regime, "Trans. of the ASME: Journal of Dynamic Systems, Measurement and Control, v.122, no.4, pp.632640, December 2000.

[8] Hung, J. Y., W. Gao and J. C. Hung, "Variable Structure Control: A Survey," IEEE Transactions on Industrial Electronics, Vol. 40, No. 1, pp. 222, February 1993.

[9] Young, K. D., V. I. Utkin, and U. Ozguner, "A Control Engineer's Guide to Sliding Mode Control", IEEE Transactions on Control Systems Technology, Vol. 7, No. 3, pp. 328-342, May 1999.

[10] Detmer, P, Bashein, G, Martin, R, "Matched Filter Identification of Left-Ventricular Endocardial Borders in Transesophageal Echocardiograms," IEEE Transactions on Medical Imaging, Vol.9, pp.396-404, 1990.

[11] Weiss, P.L., Hunter, I.W. and Kearney, R.E., "Reduction of Physiological Signal Contamination Using Linear Filter Identification," Medical and Biological Engineering and Computing, Vol.21, pp.521524, 1983.

[12] Bitmead, R. and A. Sala, "The Carpentry and Mechanics of Joint Identification and Control: Data Preparation and Iterative Control of a Sugar Cane Crushing Mill," in Lecture Notes on Iterative Identification and Control Design (Eds.) P. Albertos and A. Sala, European Science Foundation - COSY Programme, 2000.

[13] Slotine, J.-J. E. and W. Li, Applied Nonlinear Control, Prentice-Hall, 1991.

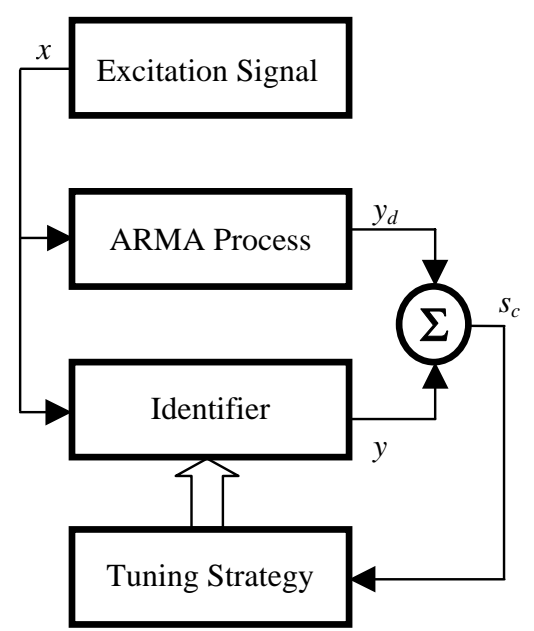

Fig. 1. Identification of a Time-Varying ARMA Process 


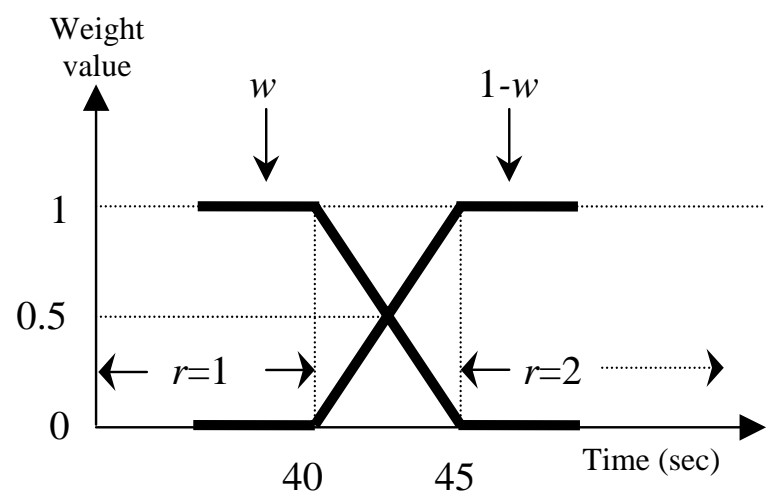

Fig. 2. The transition between intervals $r=1$ and $r=2$
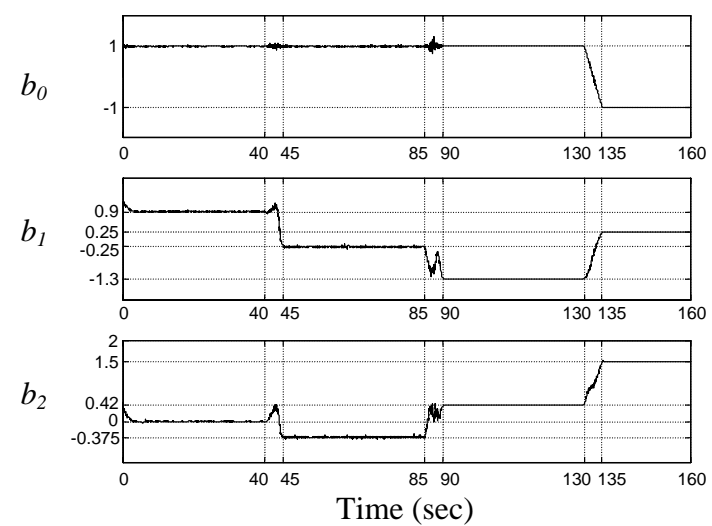

Fig. 3. Time Evolution of the Numerator Polynomial Coefficients

$a_{1}$
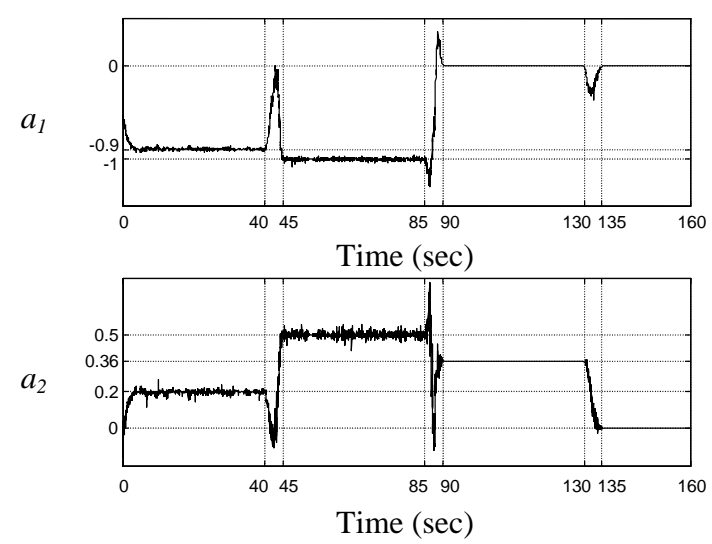

Fig. 4. Time Evolution of the Denominator Polynomial Coefficients
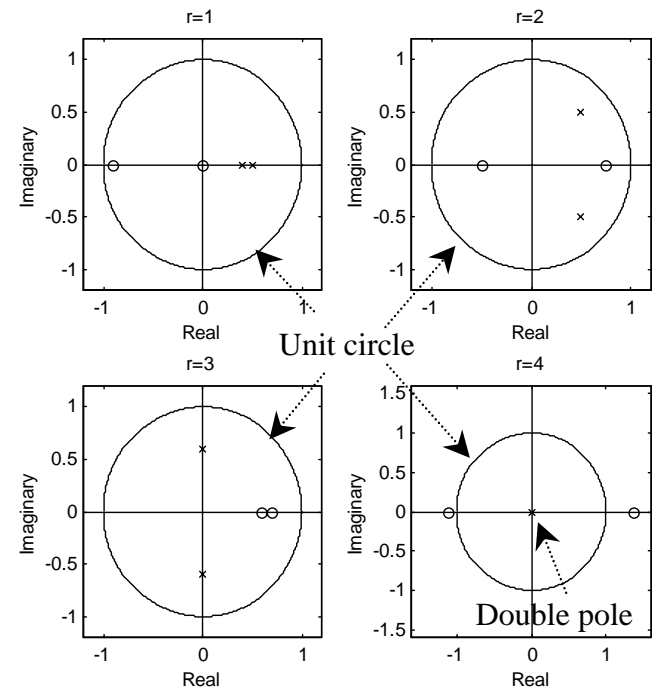

Fig. 5. Locations of the Poles and the Zeros of the Structures Activated during the Successive Intervals

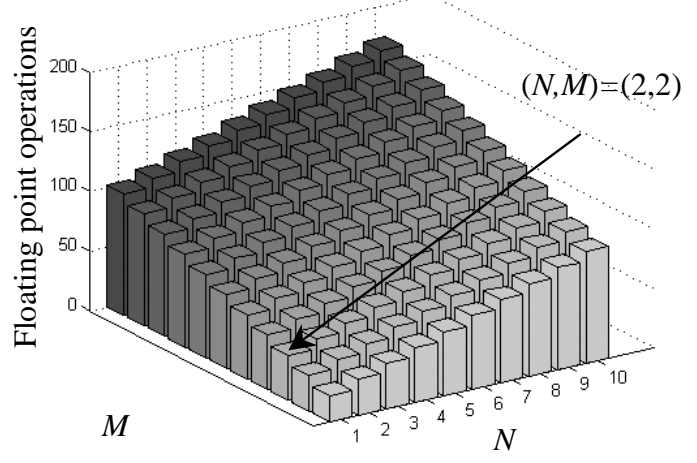

Fig. 6. Computational Requirement of the Algorithm for Varying Values of $M$ and $N$

Table 1. Parameters of the ARMA Process to be Identified

\begin{tabular}{|c|c|c|c|c|c|}
\hline Interval & \multicolumn{2}{|c|}{ Time (sec) } & $b_{0}$ & $b_{1}$ & $b_{2}$ \\
\hline$r=1$ & \multicolumn{2}{|c|}{$0 \leq \mathrm{t} \leq 40$} & 1 & 0.9 & 0 \\
\hline$r=2$ & \multicolumn{2}{|c|}{$45 \leq \mathrm{t} \leq 85$} & 1 & -0.25 & -0.375 \\
\hline$r=3$ & \multicolumn{2}{|c|}{$90 \leq \mathrm{t} \leq 130$} & 1 & -1.3 & 0.42 \\
\hline$r=4$ & \multicolumn{2}{|c|}{$135 \leq \mathrm{t} \leq 160$} & -1 & 0.25 & 1.5 \\
\hline \multicolumn{2}{|c|}{ Interval } & \multicolumn{2}{|c|}{ Time (sec) } & $a_{1}$ & $a_{2}$ \\
\hline \multicolumn{2}{|c|}{$r=1$} & \multicolumn{2}{|c|}{$0 \leq \mathrm{t} \leq 40$} & -0.9 & 0.2 \\
\hline \multicolumn{2}{|c|}{$r=2$} & \multicolumn{2}{|c|}{$45 \leq t \leq 85$} & -1 & 0.5 \\
\hline \multicolumn{2}{|c|}{$r=3$} & \multicolumn{2}{|c|}{$90 \leq \mathrm{t} \leq 130$} & 0 & 0.36 \\
\hline \multicolumn{2}{|c|}{$r=4$} & \multicolumn{2}{|c|}{$135 \leq t \leq 160$} & 0 & 0 \\
\hline
\end{tabular}

